\title{
Kasuistiken
}

Ophthalmologe 2022 $\cdot 119: 388-391$

https://doi.org/10.1007/s00347-021-01359-8

Eingegangen: 9. Februar 2021

Überarbeitet: 16. Februar 2021

Angenommen: 17. Februar 2021

Online publiziert: 16. März 2021

(c) Der/die Autor(en) 2021

\author{
V. Schöneberger ${ }^{1}$ (D) $\cdot$ F. I. Meyer ${ }^{2} \cdot$ P. Lohneis ${ }^{3} \cdot$ L. M. Heindl ${ }^{1,4}$ \\ 'Zentrum für Augenheilkunde, Medizinische Fakultät und Uniklinik Köln, Universität zu Köln, Köln, \\ Deutschland \\ ${ }^{2}$ Klinik für Radiologie, Medizinische Fakultät und Uniklinik Köln, Universität zu Köln, Köln, Deutschland \\ ${ }^{3}$ Pathologisches Institut, Medizinische Fakultät und Uniklinik Köln, Universität zu Köln, Köln, Deutschland \\ ${ }^{4}$ Centrum für Integrierte Onkologie (CIO) Aachen-Bonn-Köln-Düsseldorf, Köln, Deutschland
}

\section{Follikuläres Lymphom der Bindehaut mit aggressiv transformiertem systemischem Befall}

te eine gemeinsame Fallbesprechung in der örtlichen Tumorkonferenz stattfinden [3].

Wir möchten im Folgenden einen seltenen Fall eines follikulären Lymphoms der Konjunktiva mit aggressiver molekularer Transformation und systemischer Beteiligung präsentieren.

\section{Kasuistik}

Eine 55-jährige Patientin stellte sich vor drei Jahren erstmalig mit einer schmerzlosen, juckenden Schwellung am linken Auge vor, die seit einigen Monaten zunehmend war. Eine B-Symptomatik wurde verneint. In der Untersuchung mittels Spaltlampe zeigte sich am linken Auge eine lachsfarbene, leicht erhabene Läsion an der medialen, karunkelnahen Bindehaut (-Abb. 1 und 2). Der Vorderabschnitt war reizfrei, die bestkorrigierte Sehschärfe betrug 1,0 beidseits.

\section{Weiteres Prozedere}

Zur weiterführenden Diagnostik wurde zeitnah eine chirurgische Biopsie geplant. Die histopathologische Diagnostik nach der Plica-Exzision erbrachte ein B-NHL vom Typ eines follikulären Lymphoms Grad 1-2 der Konjunktiva mit einem Ki67-Proliferationsindex von 10-15\%.

Zur Abgrenzung eines primär konjunktivalen Lymphoms oder einer konjunktivalen Manifestation eines nodalen Lymphoms erfolgte eine klinischhämatologische Untersuchung in der onkologischen Ambulanz. Das radiologische Staging mittels Computertomographie (CT) ergab ein follikuläres Lymphom Grad 1-2 im Stadium IV A E, aufgrund eines disseminierten Lymphknotenbefalls und einer massiven $\mathrm{He}$ patosplenomegalie mit einer Milzgröße von $10 \times 16 \times 19 \mathrm{~cm}$ (normalerweise $4 \times 7 \times 11 \mathrm{~cm}$; - Abb. 3a, b), fehlender junktivalen Lymphomen die Low-GradeFormen (• Tab. 1).

Das niedrigmaligne NHL der Bindehaut liegt meist isoliert vor (76-90\%) und kann meist durch eine Bestrahlung geheilt werden [2]. Nach Diagnosestellung erfolgt die Vorstellung bei einem Onkologen zum weiteren Staging und Ausschluss einer systemischen Beteiligung, welche bei High-Grade-Lymphomen häufiger zu beobachten ist [1]. Aufgrund der erforderlichen interdisziplinären Zusammenarbeit zwischen Augenklinik, Radiologie, Hämatoonkologie und Pathologie soll-

Tab. 1 Epidemiologie der histologischen Subtypen von konjunktivalen Lymphomen. (Mod. nach Tanenbaum et al., Classification, diagnosis, and management of conjunctival lymphoma (2019); [2])

\begin{tabular}{lll} 
Histologie & Grading & $\begin{array}{l}\text { Anteil der konjunktivalen Lym- } \\
\text { phome (\%) }\end{array}$ \\
\hline $\begin{array}{l}\text { Extranodales Marginalzonenlym- } \\
\text { phom }\end{array}$ & Low-Grade-Tumor & 80 \\
$\begin{array}{l}\text { Follikuläres Lymphom } \\
\text { Diffus großzelliges B-Zell-Lymphom }\end{array}$ & Low-Grade-Tumor & 8 \\
Mantelzelllymphom & High-Grade-Tumor & 3 \\
\hline T-Zell-Non-Hodgkin-Lymphom & High-Grade-Tumor & 3 \\
\hline High-Grade-Tumor & 2
\end{tabular}




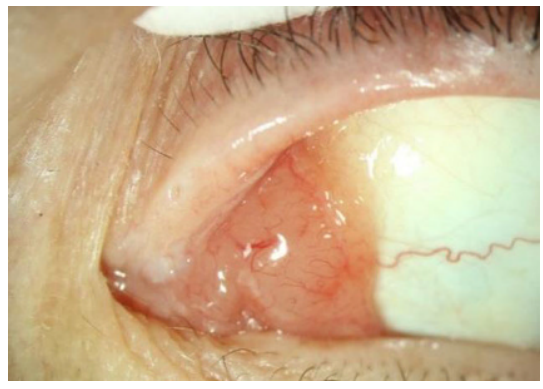

Abb. $1 \Delta$ Befund bei Erstvorstellung. Lachsfarbene, erhabene Läsion im Bereich der medialen, karunkelnahen Konjunktiva des linken Auges

B-Symptomatik (A) und extranodalem Befall (E) der Konjunktiva. Die Histologie der konjunktivalen Biopsie war ausreichend, um eine systemische Therapie mittels R-CHOP (Rituximab, Cyclophosphamid, Doxorubicin, Vincristin und Prednisolon) einzuleiten.

Jedoch wurde die Patientin nur wenige Tage später mit ausgeprägter B-Symptomatik (Fieber, Schüttelfrost, Tachykardien) stationär in einer externen Uniklinik aufgenommen. Dort erfolgte eine CT-gesteuerte Punktion eines iliakalen Lymphknotens (• Abb. 3c) und des Knochenmarks. Daraus ergab sich die Diagnose eines aggressiven NHL vom Typ des diffus großzelligen B-ZellLymphoms bei Ann-Arbor IV B (B-Symptomatik). Die immunhistochemischen Färbungen zeigten nun im Gegensatz zur Probebiopsie der Konjunktiva eine Negativität für bcl2 und CD10. Bei Expression von BCL6 und MUM1 handelte es sich nach der Hans-Klassifikation [5] um einen Non-GCB(Germinal-Center-B-Cell-like)-Typ (• Abb. 4b-d). Es erfolgte eine Vorphasentherapie mit Cyclophosphamid und Dexamethason mit anschließender Therapie nach dem R-CHOP-Schema. In der Verlaufs-CT konnte daraufhin eine deutliche Regredienz der bekannten Lymphommanifestationen, einschließlich des okulären Befundes, festgestellt werden.

\section{Diskussion: sekundär simultanes DLBCL oder primär transformiertes follikuläres Lymphom?}

Sowohl beim diffus großzelligen B-ZellLymphom (DLBCL) als auch beim trans- formierten follikulären Lymphom (t-FL) handelt es sich um aggressive Lymphome, ganz im Gegensatz zum indolenten follikulären Lymphom. Im Allgemeinen ist das follikuläre Lymphom das zweithäufigste diagnostizierte Lymphom in den Vereinigten Staaten und Westeuropa und transformiert in etwa $22 \%$ innerhalb von 5 Jahren. $\mathrm{Zu}$ den follikulären Lymphomen der okulären Adnexen liegen nur wenige Daten vor. Rasmussen et al. beschrieben in einer internationalen multizentrischen retrospektiven Studie über einen Zeitraum von 30 Jahren lediglich eine Transformationsrate von $3 \%$ bei follikulären Lymphomen der okulären Adnexe. Eine Transformation geht einher mit einer schnelleren Progression der Lymphadenopathie, B-Symptomen, erhöhtem LDH-Wert, Hyperkalzämie und einem Nichtansprechen auf die Initialtherapie [6-9].

Follikuläre Lymphome leiten sich von B-Zellen des Keimzentrums („germinal centerB-cells“, GCB) ab. Daher ist es nicht verwunderlich, dass die allermeisten $\mathrm{t}-\mathrm{FL}$ einen GCB-Phänotyp haben (89\%) [5]. Allgemein kann ein t-FL morphologisch nicht von einem De-novo-DLBCL unterschieden werden [10]. In unserem Fall wurde daher zunächst bei vorliegendem Non-GCB-Typ und der schnellen Progression von einer zweiten, unabhängigen Lymphommanifestation in kürzestem Zeitraum ausgegangen. Im Gegensatz zu einem transformierten Lymphom ist das Ansprechen auf eine Chemotherapie bei einer De-novo-Erkrankung besser und die Überlebenszeit länger [11].

Um die Frage abschließend zu klären, ob es sich um ein sekundär simultanes DLBCL oder primär transformiertes follikuläres Lymphom handelt, wurde eine IgH-Klonalitätsanalyse durchgeführt. Die Analyse beruht methodisch auf einer PCR-Fragmentanalyse und zeigt spezifische Rearrangements in der leichten und schweren Kette des Immunglobulin-GGens. Dadurch kann die Abstammung von einer gemeinsamen Ursprungszelle (Klonalität) molekularpathologisch festgestellt werden. Die Untersuchung zeigte überraschenderweise übereinstimmende DNA-Fragmentlängen in der Probebiopsie der Karunkula und des iliakalen Lymphknotens, wodurch schlussendlich die Diagnose eines tFL vom selteneren ABC-Typ getroffen werden konnte.

Dieser Fall zeigt auf, dass die $\mathrm{Zu}$ ordnung zur Ursprungszelle („cell of origin“) wichtig ist, um eine klare Diagnose zu treffen und entsprechend auch die Prognose und das Ansprechen auf eine zielgerichtete Therapie vorhersagen zu können. Aktuell sieht man keine schlechtere Prognose beim ABC-Typ im Vergleich zum GCB-Typ, jedoch liegen aufgrund der Seltenheit des Befundes nur wenige Daten vor [10]. Die Feststellung, dass eine Untergruppe der t-FL dem ABC-Subtyp angehört, ist besonders faszinierend, da sie die Frage aufwirft, ob diese Patienten überhaupt Kandidaten für eine gezielte Beeinflussung des B-Zell-Rezeptor-Signalwegs wären. So könnten zielgerichtete Wirkstoffe wie Ibrutinib und Lenalidomid bei ABC-tFL untersucht werden. Aktuell läuft eine Phase II-Studie bei refraktären oder rezidivierten DCBLC mit Non-GCB-Typ (registriert unter \#NCT02077166) [12].

\section{Weiterer Verlauf}

Ende des letzten Jahres stellte sich uns die Patientin erneut bei dringlichem Verdacht auf ein Lymphomrezidiv intraorbital links vor. In der CT waren eine orbitale Raumforderung links (• Abb.5) und geschwollene Lymphknoten mediastinal und rechts axillär zu sehen. Eine axilläre Lymphknotenbiopsie erbrachte jedoch einen negativen Befund.

Durch die ausgeprägte orbitale Raumforderung kam es bei der Patientin zu einem Exophthalmus und einer Exophorie mit zunehmenden Doppelbildern. Der Visus verschlechterte sich linksseitig bestkorrigiert auf 0,4. Die inzisionale Biopsie mittels eines transkonjunktivalen anterioren Zugangs erbrachte nach histologischer und molekularbiologischer Untersuchung erneut den Befund eines niedrigmalignen, follikulären NHL der $B$-Zellreihe, das als Rezidiv des bekannten follikulären Lymphoms drei Jahre zuvor $\mathrm{zu}$ werten war. Es erfolgte umgehend die Einleitung einer Rezidivtherapie mit Rituximab und Bendamustin. Ein halbes Jahr später erfolgte ein CT-Staging, das eine erhebliche Größenregredienz aller vorbekannten Lymphommanifes- 


\section{Kasuistiken}
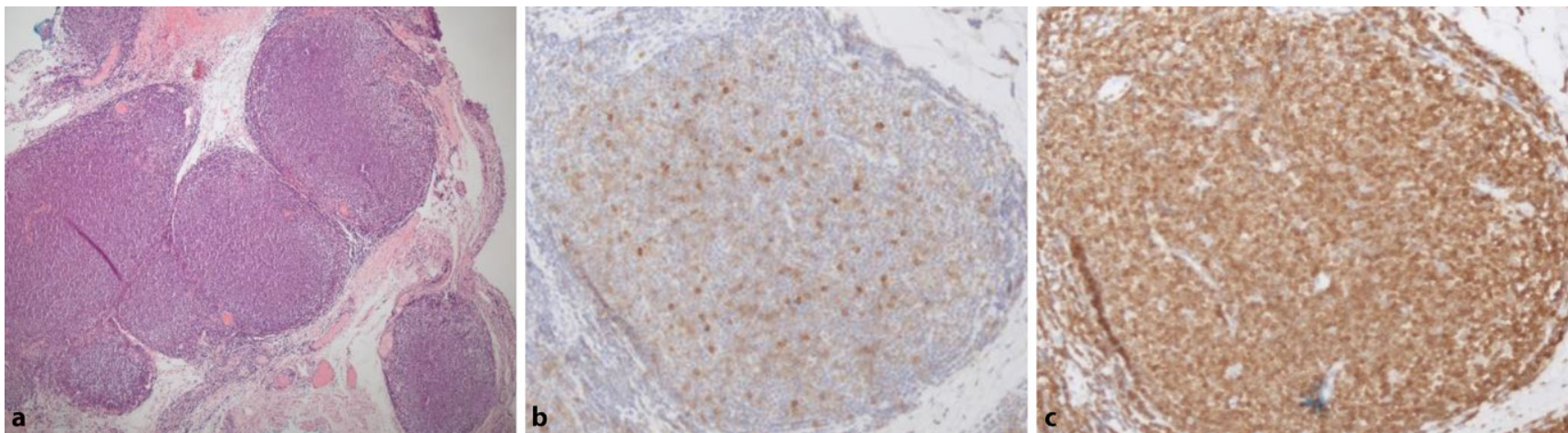

Abb. $2 \Delta$ Histologische Färbungen der chirurgischen Biopsie links. Follikuläres Lymphom. a Hämatoxylin-Eosin-Färbung der Probebiopsie. In der Übersichtsvergrößerung erkennt man follikulär aufgebautes lymphatisches Gewebe. b Die Lymphozyten sind positiv für CD10 c und zeigen als Hinweis auf eine Translokation $(14 ; 18)$ eine deutliche bcl2-Expression
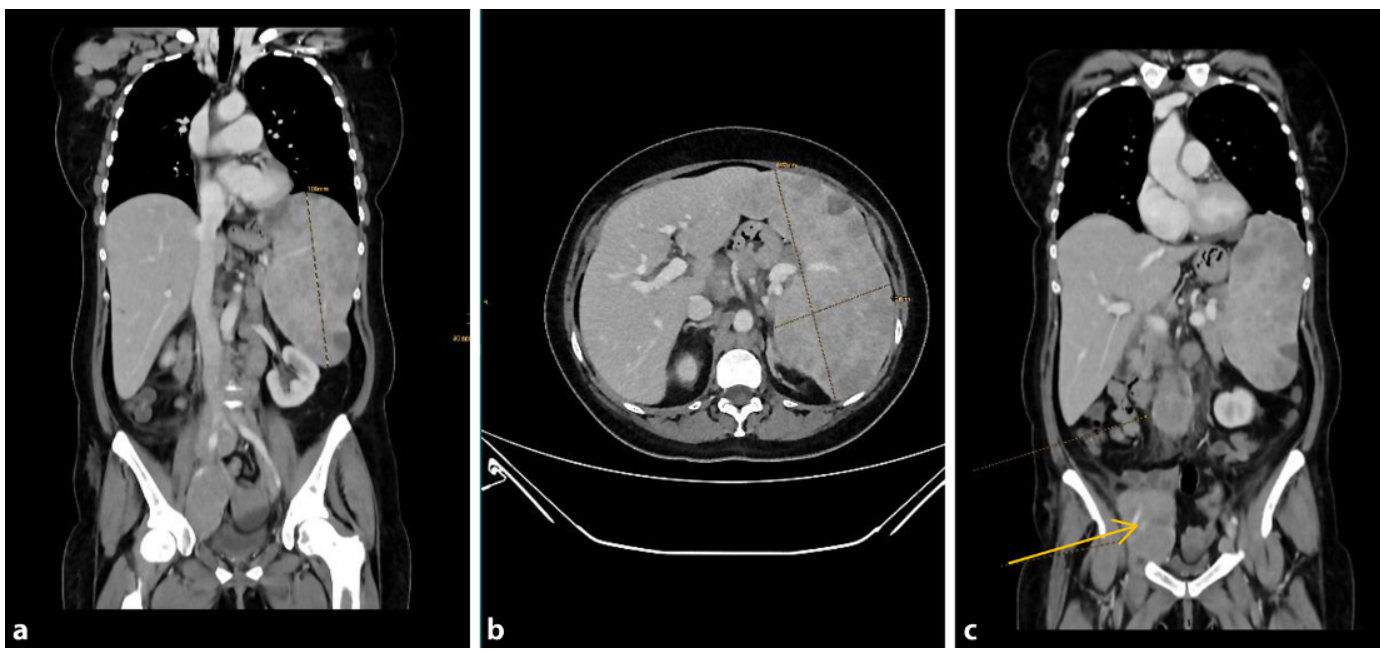

Abb. $3<$ CT-Staging, Beispielbilder $\mathbf{a}$ und $\mathbf{b}$ mit ausgeprägter Splenomegalie $(10 \times 16 \times 19 \mathrm{~cm})$ und Hepatomegalie, c massive iliakale Raumforderung rechtsseitig
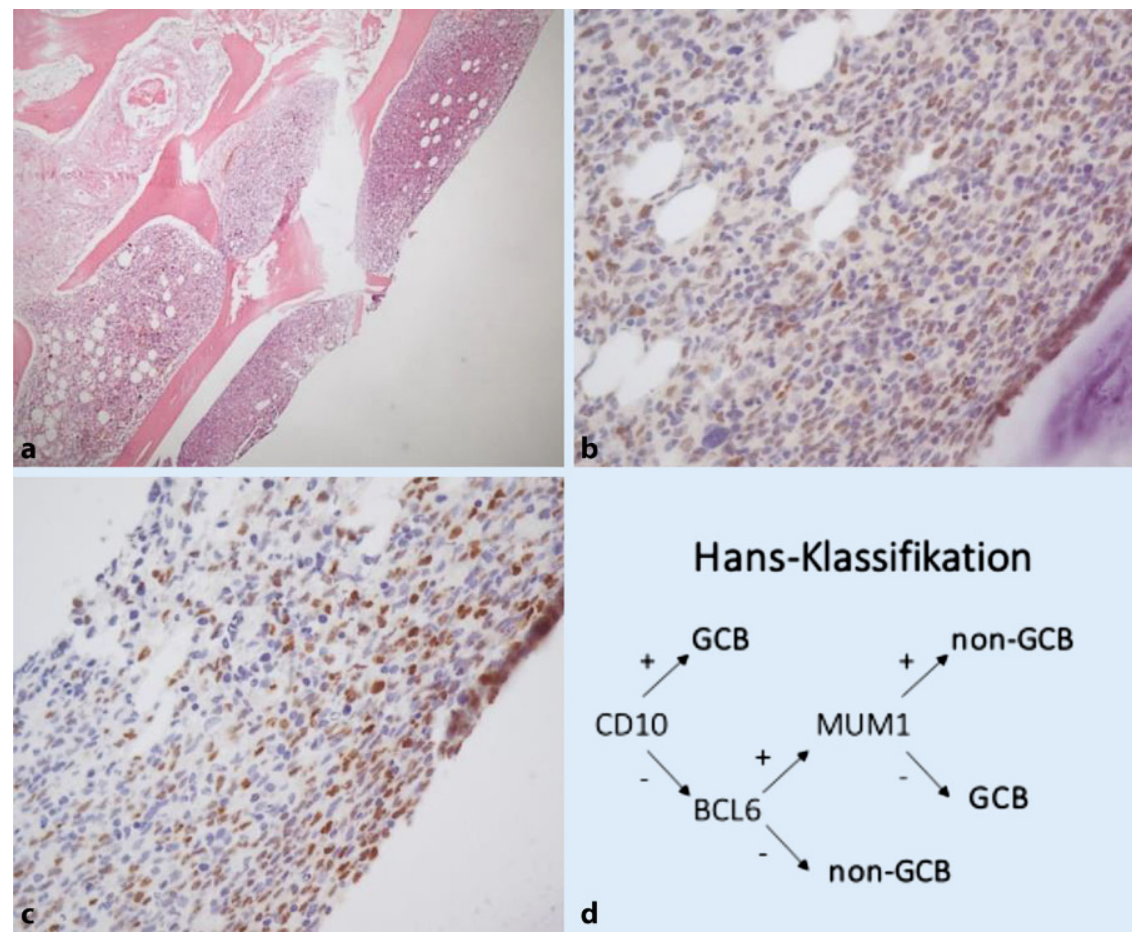

Abb. 4 \ Knochenmarkmanifestation des diffus großzelligen B-Zell-Lymphoms. a Hämatoxylin-Eosin-Färbung, b etwa $60 \%$ der Zellen mäßig stark nukleär positiv für BCL6, c deutlich positiv für MUM1, d Schema der Hans-Klassifikation 

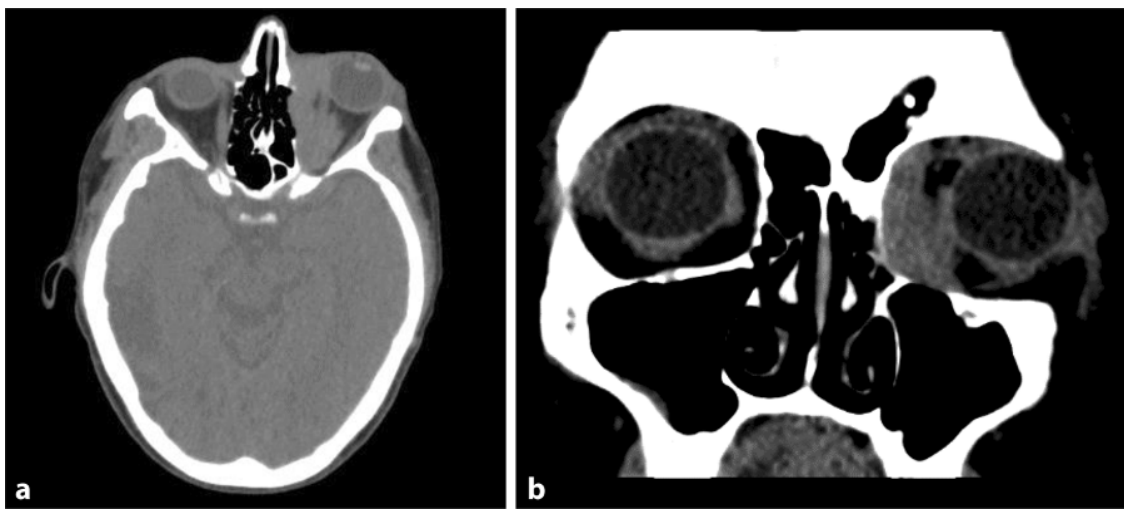

Abb. 5 ॥ Schädel-CT, extern durchgeführt, mit Kontrastmittel. Orbitale Raumforderung links, a Transversalschnitt, b Koronarschnitt

tationen zeigte. Die Patientin befindet sich weiterhin in der Tumornachsorge, aktuell gehe es ihr gut.

\section{Fazit für die Praxis}

- Die Transformation eines Lymphoms ist selten, unter Beteiligung des Auges sogar ungewöhnlich.

- Hier wird ein solcher Fall mit aggressiver histologischer Transformation von einem ursprünglichen (indolenten) Low-Grade-Lymphom beschrieben.

- Eine Transformation eines indolenten Lymphoms in ein aggressives Lymphom geht mit einer schlechteren Prognose einher.

\section{Korrespondenzadresse}

Dr. med. V. Schöneberger
Zentrum für Augenheilkunde,
Medizinische Fakultät und
Uniklinik Köln, Universität zu
Köln
Kerpener Str. 62, 50937 Köln,
Deutschland
verena.schoeneberger@
uk-koeln.de

Funding. Open Access funding enabled and organized by Projekt DEAL.

\section{Einhaltung ethischer Richtlinien}

Interessenkonflikt. V. Schöneberger, F.I. Meyer, P. Lohneis und L.M. Heindl geben an, dass kein Interessenkonflikt besteht.>

Für diesen Beitrag wurden von den Autoren keine Studien an Menschen oder Tieren durchgeführt. Für die aufgeführten Studien gelten die jeweils dort ange- gebenen ethischen Richtlinien. Für Bildmaterial oder anderweitige Angaben innerhalb des Manuskripts, über die Patienten zu identifizieren sind, liegt von ihnen und/oder ihren gesetzlichen Vertretern eine schriftliche Einwilligung vor.

Open Access. Dieser Artikel wird unter der Creative Commons Namensnennung 4.0 International Lizenz veröffentlicht, welche die Nutzung, Vervielfältigung, Bearbeitung, Verbreitung und Wiedergabe in jeglichem Medium und Format erlaubt, sofern Sie den/die ursprünglichen Autor(en) und die Quelle ordnungsgemäß nennen, einen Link zur Creative Commons Lizenz beifügen und angeben, ob Änderungen vorgenommen wurden.

Die in diesem Artikel enthaltenen Bilder und sonstiges Drittmaterial unterliegen ebenfalls der genannten Creative Commons Lizenz, sofern sich aus der Abbildungslegende nichts anderes ergibt. Sofern das betreffende Material nicht unter der genannten Creative Commons Lizenz steht und die betreffende Handlung nicht nach gesetzlichen Vorschriften erlaubt ist, ist für die oben aufgeführten Weiterverwendungen des Materials die Einwilligung des jeweiligen Rechteinhabers einzuholen.

Weitere Details zur Lizenz entnehmen Sie bitte der Lizenzinformation auf http://creativecommons.org/ licenses/by/4.0/deed.de.

\section{Literatur}

1. Kirkegaard MMetal (2015) Malignantlymphoma of the conjunctiva. Surv Ophthalmol 60(5):444-458

2. TanenbaumREetal(2019)Classification, diagnosis, and management of conjunctival lymphoma. Eye Vis (Lond) 6:22

3. Kakkassery V et al (2020) Ocular lymphoma: precise diagnostics and classification as key for successful personalized treatment. Ophthalmologe 117(6):499-507

4. Kakkassery $\mathrm{V}$ et al (2015) Lymphoma of the ocular adnexa. Ophthalmologe 112(3):210-216

5. Hans CP et al (2004) Confirmation of the molecular classification of diffuse large B-cell lymphoma by immunohistochemistry using a tissue microarray. Blood 103(1):275-282

6. Freedman AS (2005) Biology and management of histologic transformation of indolent lymphoma. Hematology Am Soc Hematol Educ Program 2005(1):314-320
7. Swerdlow SH et al (2016) The 2016 revision of the World Health Organization classification of lymphoid neoplasms. Blood 127(20):2375-2390

8. Bastion $Y$ et al (1997) Incidence, predictive factors, and outcome of lymphoma transformation in follicular lymphoma patients. J Clin Oncol 15(4):1587-1594

9. Rasmussen PKet al (2014) Ocular adnexal follicular lymphoma: a multicenter international study. JAMA Ophthalmol 132(7):851-858

10. Kridel $\mathrm{R}$ et al (2015) Cell of origin of transformed follicular lymphoma. Blood 126(18):2118-2127

11. Davies AJ (2007) Radioimmunotherapy for B-cell lymphoma: Y90 ibritumomab tiuxetan and I(131) tositumomab. Oncogene 26(25):3614-3628

12. Goy A et al (2019) Ibrutinib plus lenalidomide and rituximab has promising activity in relapsed/ refractory non-germinal center B-cell-like DLBCL. Blood 134(13):1024-1036 